\title{
Next Generation Hole Injection/Transport Nano-Composites for High Efficiency OLED Development
}

\author{
Sponsored by \\ Solid State Lighting Core Technology Program \\ Department of Energy
}

Agiltron, Inc.

Final Technical Report

September 30, 2007

\section{Contract No. DE-FC26-06NT42858}

Principal Investigator: $\quad$ Dr. King Wang

Effective date of Contract: $\quad$ 08/01/06

Contract Expiration Date: 07/31/09

Total Dollar Value: $\quad \$ 1,376,366.00$

The views, opinions, and findings contained in this report are those of the author(s) and should not be construed as an official Department of Energy position, policy, or decision, unless so designated by other pfficial documentation.

AGILTRON INC.

15 Cabot Road

Woburn, MA 01801

781-935 1200

www.agiltron.com 


\section{DISCLAIMER}

This report was prepared as an account of work sponsored by an agency of the United States Government. Neither the United States Government nor any agency thereof, nor any of their employees, makes any warranty, express or implied, or assumes any legal liability or responsibility for the accuracy, completeness, or usefulness of any information, apparatus, product, or process disclosed, or represents that its use would not infringe privately owned rights. Reference herein to any specific commercial product, process, or service by trade name, trademark, manufacturer, or otherwise does not necessarily constitute or imply its endorsement, recommendation, or favoring by the United States Government or any agency thereof. The views and opinions of authors expressed herein do not necessarily state or reflect those of the United States Government or any agency thereof. 


\section{Project Objectives}

The objective of this program is to use a novel nano-composite material system for the OLED anode coating/hole transport layer. The novel anode coating is intended to significantly increase not only hole injection/transport efficiency, but the device energy efficiency as well. Another goal of the Core Technologies Program is the optimization and scale-up of air-stable and cross-linkable novel HTL nano-composite materials synthesis and the development of low-cost, large-scale mist deposition processes for polymer OLED fabrication. This proposed technology holds the promise to substantially improve OLED energy efficiency and lifetime.

\section{Project Accomplishment Summary}

This Core Technology project leverages Agiltron's Phase II achievement and further enhances the hole injection performance of hole transport materials including Agiltron's hole transport materials (AG I and II), and other commercial hole transport polymers as well. In the Phase II project, Agiltron has successfully synthesized cross-linkable hole transport materials (AG I and AG II) and demonstrated these materials possess excellent hole transport and electron blocking properties. We demonstrated that AG materials can work together with PEDOT as an electron blocking material and achieved a 40\% power efficiency improvement.

In this Core Technology project, Agiltron proposed a nano-composite approach to further enhance hole injection performance of Agiltron's hole transport materials and commercial hole transport polymers as well. In this program, Agiltron has already successfully demonstrated the feasibility of the proposed hole injection enhancement. The enhanced hole injection stems from concentrated electric filed on the top of nanoparticles. We have also clearly demonstrated that the proposed nano-composite structures can be made using a novel and proprietary mist coating process, indicating low cost potential for polymer LEDs to meet DOE cost targets for Solid State Lighting Products. Based on these inventions, Agiltron believes that it has made some of the most significant progress to date in this exciting technology area.

\section{Work Performed in This Program}

\section{Fabrication of Nano-Composite Hole Injection/Transport Layers}

\section{(A). “Two-Step” Approach}

We employed a "Two Step" approach to make the proposed nano-composite hole injection/transport layers. First, nano-particles are assembled on an ITO substrate, then a hole transport polymer layer is spin-coated on the nano-structured substrates. In order to achieve energy level matching between nano-particles and the substrate, ITO and Au nano-particles are selected. 
Commercial ITO nano-powder was used with a particle size of $\sim 20 \mathrm{~nm}$. The ITO nanoparticles (NPs) were dispersed in ethanol with the help of carboxylic acid as a dispersion agent, and negatively charged. Commercial negatively charged Au nano-particles colloid with a particle size of $\sim 10 \mathrm{~nm}$ was selected for Au NP self-assembly. The starting materials are shown in Figure 1.

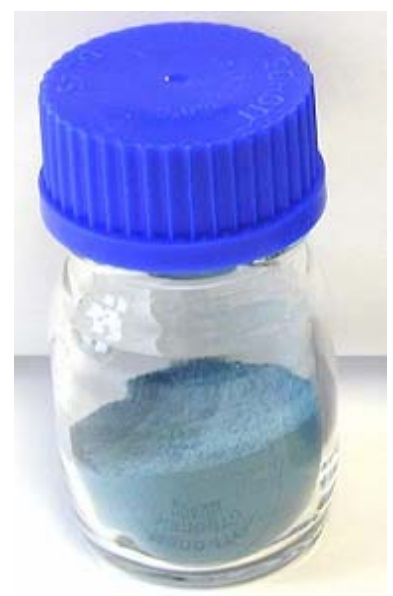

(a)

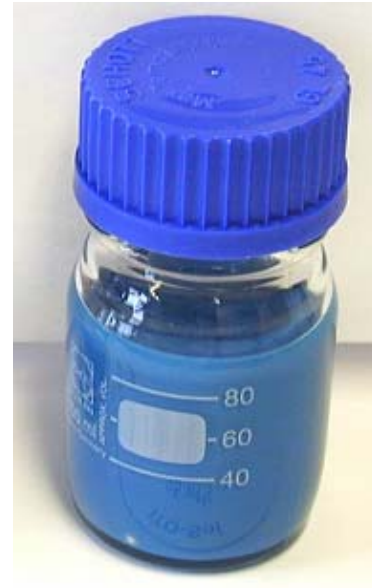

(b)

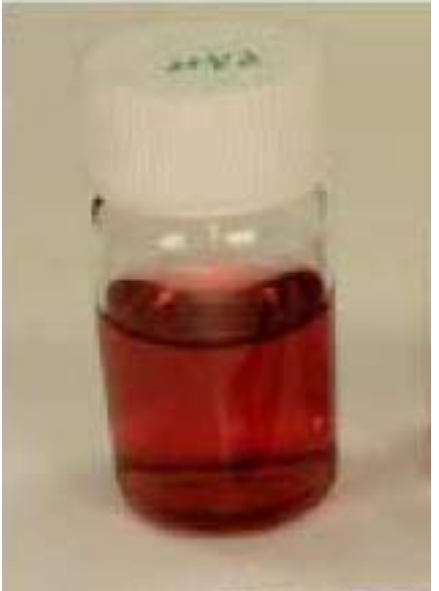

(c)

Figure 1: (a) Commercial ITO nano-powder; (b) Well dispersed ITO nano-particle colloid; and, (c) Commercial Au nano-particle colloid.

We self assembled a monolayer of ITO NPs on ITO coated glass substrates. ITO coated glass substrate was firstly degreased. Then it was immersed in an electrolyte for positive charges. A positively charged surface is obtained after rinse and dry. Negatively charged ITO nanoparticles were self-assembled onto ITO substrates via electrostatic attraction.

We also coated the gold nanoparticles on the surface of ITO coated glass substrates through spin-coating and self-assembly monolayer method. The gold nanoparticles (10 $\mathrm{nm})$ were purchased from Sigma Aldrich. The same procedures as described above were adopted to prepare the electrodes.

The above spin-coated and self-assembled samples were treated in an oven at $350{ }^{\circ} \mathrm{C}$ for $4 \mathrm{~h}$ in air, after which they are treated in oven at $350{ }^{\circ} \mathrm{C}$ for another $4 \mathrm{~h}$ in atmosphere of $\mathrm{N}_{2}: \mathrm{H}_{2}=95: 5$. Figure 2 shows the two treated Au (left) and ITO (right) NP coated ITO substrates. 


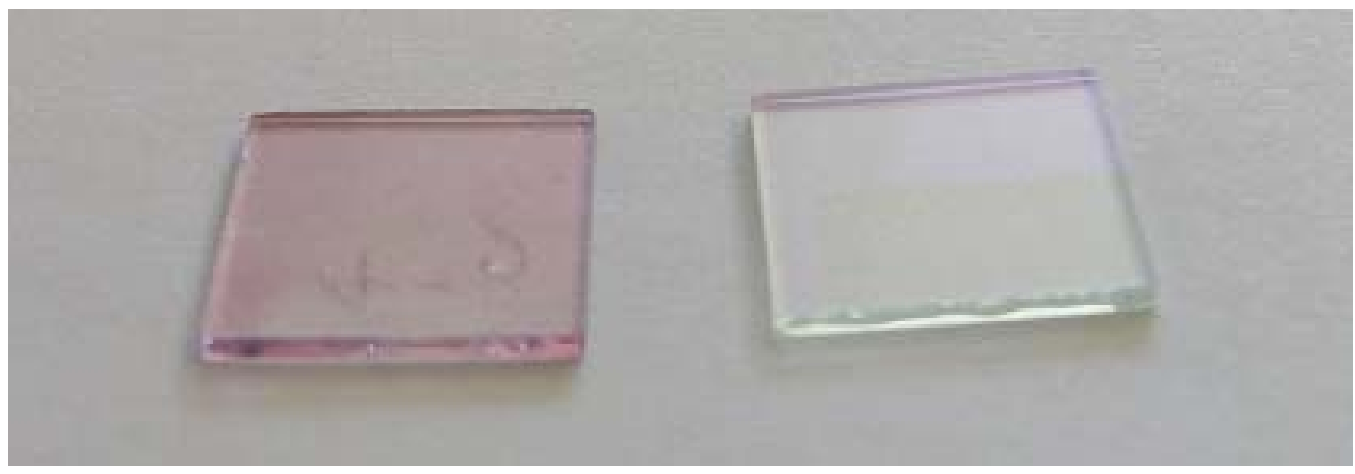

Figure 2: Au (left) and ITO (right) NP coated ITO substrates.

We have characterized the micro-structures of the NP coated substrates using AFM, as shown in Figure 3. Their RMS roughnesses are $2.173 \mathrm{~nm}$ and $2.838 \mathrm{~nm}$ for $\mathrm{Au}$ and ITO NP coated surface, respectively. The surfaces of SA nano-particle coatings are smooth enough for OLED fabrication. Highly efficient hole injection can be expected from these nano-structures.
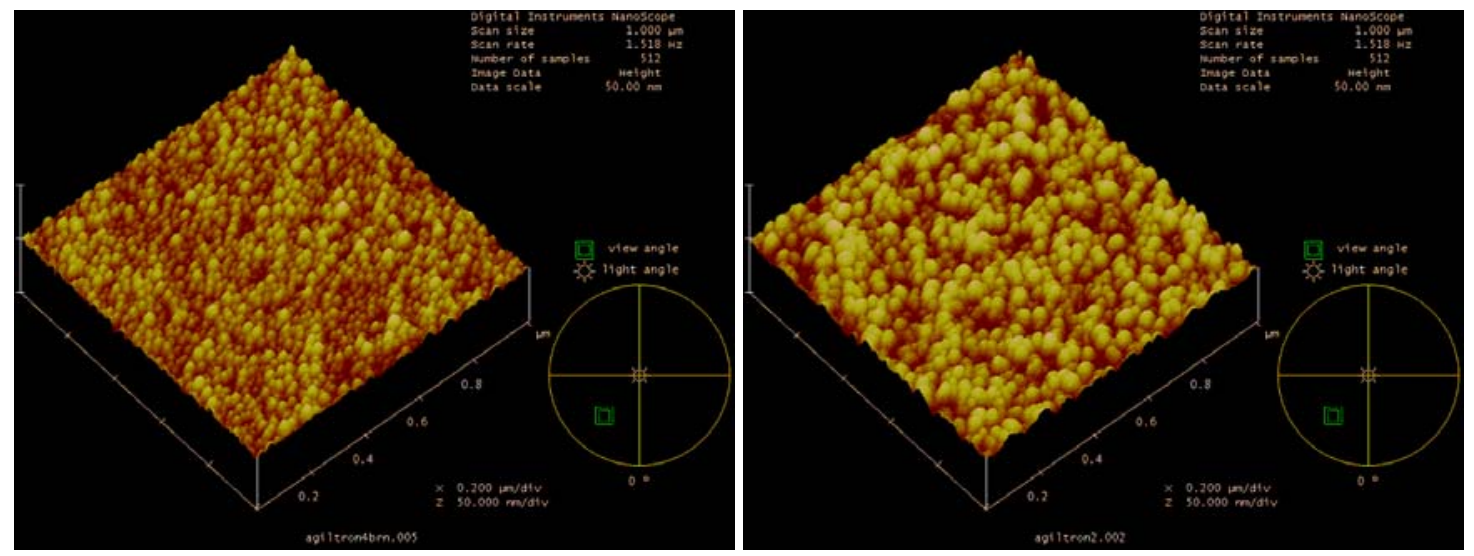

Figure 3: AFM images of Au (left) and ITO (right) NP coated ITO substrates.

In order to make the nano-composite hole transport layer, a hole transport polymer can be spin coated on the nano-structured surface. In this program, we only tried PEDOT as a hole transport polymer. Other hole transport polymers will be used in our future research and compared with PEDOT in the proposed nano-composite systems.

\section{(B). “One-Step” Approach}

One-Step approach means mixing dispersed nano-particles with hole transport polymers first followed by spin-coating the nano-particle dispersion onto substrates. We tried different dispersants and found that phosphoric acid is the best dispersant of ITO powder, and it is compatible with PEDOT solution. The following procedures are used to prepare stable PEDOT-ITO dispersion. 
$0.2 \mathrm{~g}$ ITO is mixed with $9 \mathrm{ml}$ ethanol and $1 \mathrm{ml}$ ethylene glycol. One drop of phosphoric acid is added to the solution. The solution is sonicated for 3 hours. 10ml PEDOT (Baytron P 4083) is added into the ITO dispersion. The mixture is sonicated for 3 hours, and pass through $0.7 \mu \mathrm{m}$ glass fiber filter. Stable PEDOT-ITO dispersion is obtained, as shown in Figure 4. The same procedure is used to prepare PEDOT-ITO dispersion without using any dispersant. However, the dispersion is less stable than PEDOT-ITO with phosphoric acid as a dispersant.

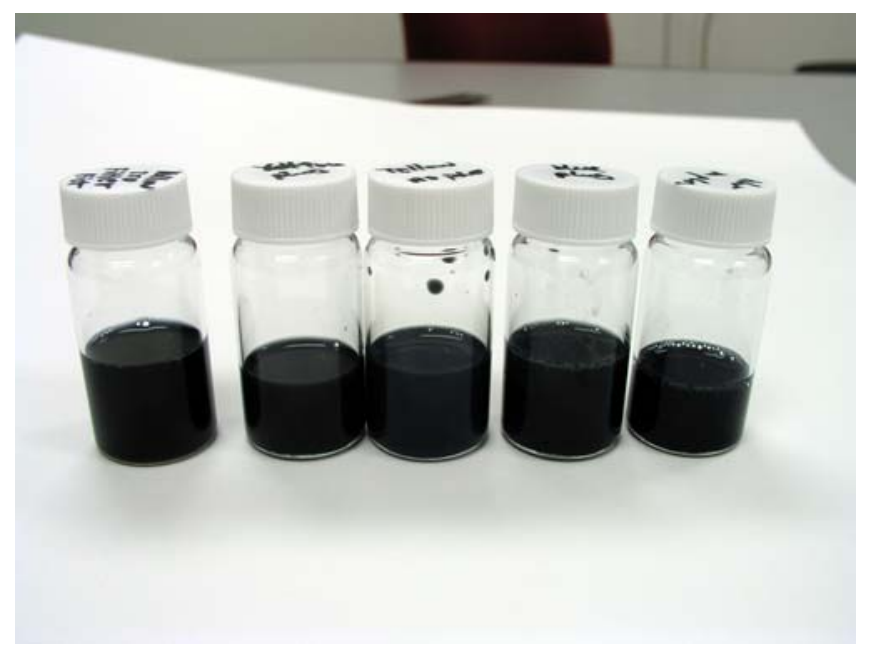

Figure 4: From left to right, PEDOT and ITO-PEDOT dispersions with different ITO concentrations.

Compared to "Two-Step" approach, "One-Step" approach has several limitations. 1), there are very limited hole transport polymers are compatible with the nano-particle dispersion. 2), the surface morphologies of nano-composite films are too rough for OLED devices, leading to large leakage current and low power efficiency. Therefore, most of the device fabrication and evaluation work in this program is based on "Two-Step" coated substrates.

\section{OLED Fabrication and Evaluation}

High power efficient OLED devices have been fabricated on the nano-composite hole transport layer, and their performance evaluated and compared with devices without this nano-composite layer. The devices were fabricated and evaluated at UCLA with Agiltron provided nano-composite layer coated substrates. The schematic device structure is shown in Figure 5 (a), with that of control device with blank substrate shown in Figure 5 (b) 

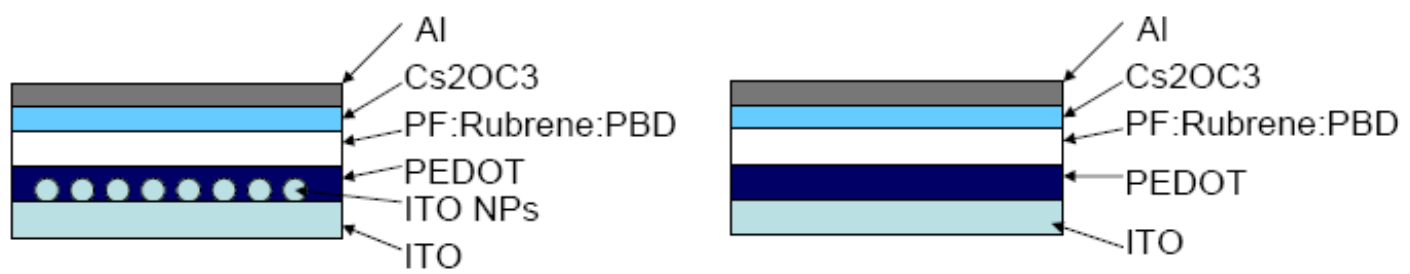

Figure 5: (a) A schematic device structure of Agiltron's OLED with nano-composite hole transport layer, (b) A schematic device structure of control device with blank substrate.

The evaluation of these devices is shown in Figure 5 . As we proposed, the application of Agiltron's hole injection material will increase the hole injection, which leads to higher current density and luminance compared to the device without Agiltron's materials, as shown in Figure 6 (a) and (b). The current device conditions, for example, electron injection ability, electron transport ability, thickness of polymer layers, are all optimized for a device without Agiltron's hole injection material. The enhanced hole injection might damage the current balance in the device due to excess hole injection, which can be the reason for the lower current efficiency, as shown in Figure 5 (c). We are planning to optimize electron injection at the cathode/emissive layer interface to achieve balanced hole/electron injection in Year II of this program, which will lead to much higher power efficiency.

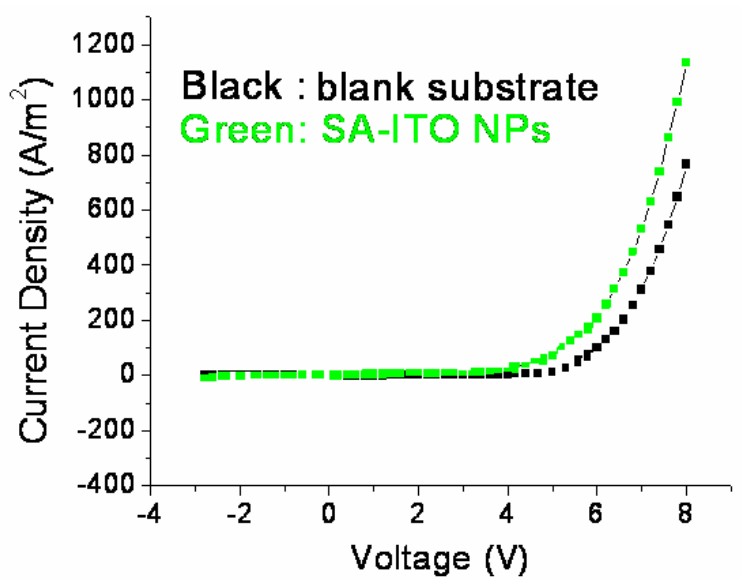

(a)

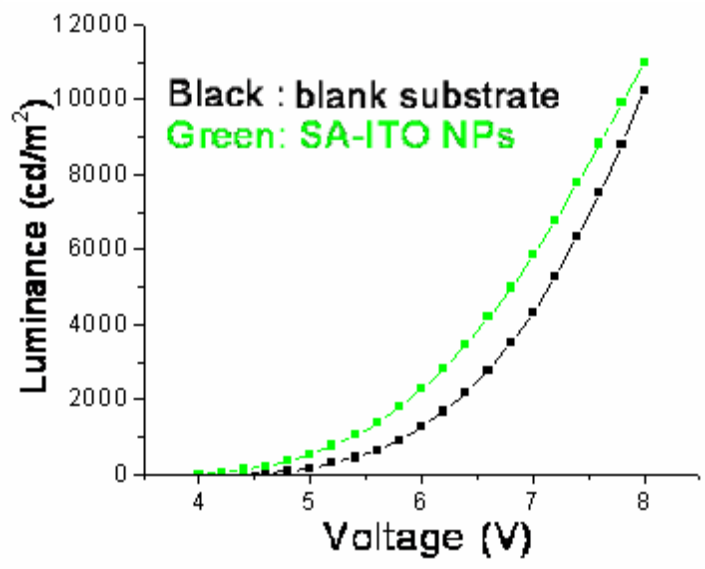

(b) 


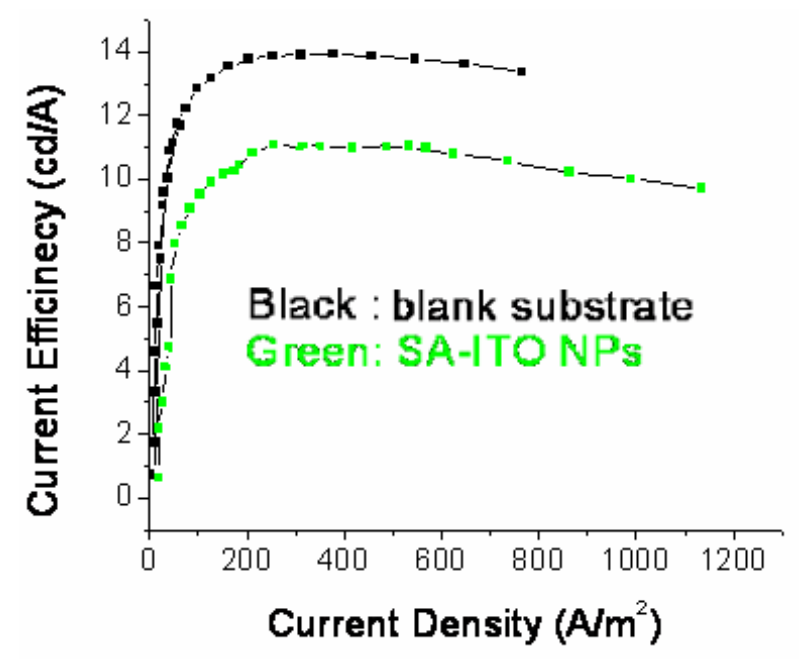

(c)

Figure 6: Current density vs voltage (a), luminance vs voltage (b), and current efficiency vs current density (c) of an Agiltron's OLED device

We have successfully demonstrated fluorescent white polymer LEDs with an energy efficiency of $18 \mathrm{~lm} / \mathrm{W}$, as shown in Figure 7. A power efficiency of $\sim 27 \mathrm{~lm} / \mathrm{W}$ can easily be achieved using standard light extraction enhancement approaches such as a micro-lens array thus achieving our goal for the first year. White light has an excellent color rendering index of over 75, as shown in Figure 8. The device structure needs to be optimized for the coated substrate to achieve higher power efficiency. Extensive device fabrication and evaluation are being performed.

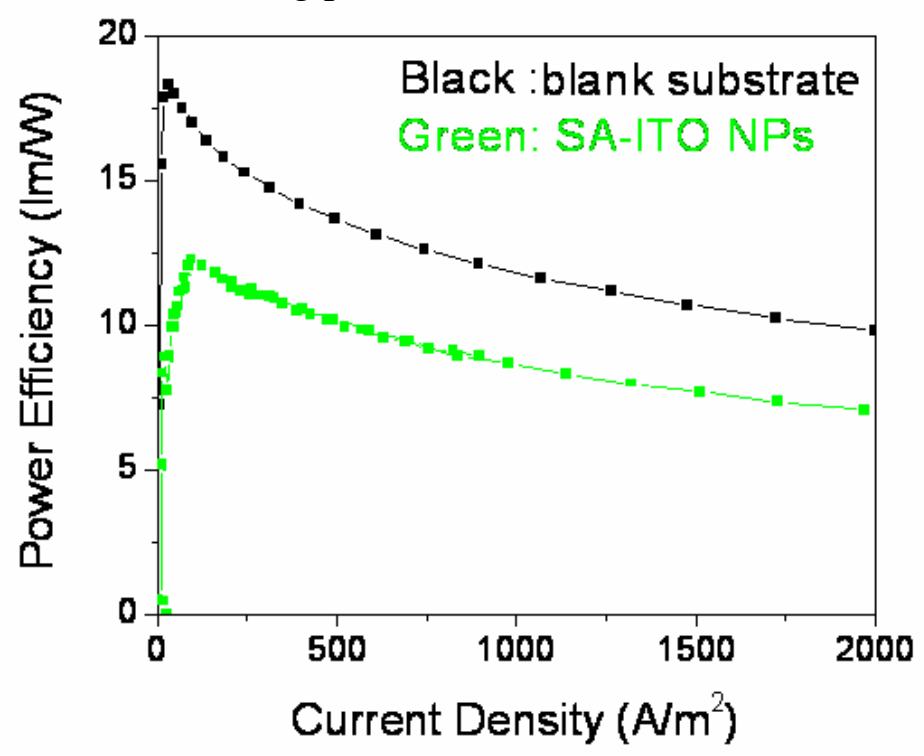

Figure 7: Power efficiency vs current density of Agiltron's white OLED devices. 


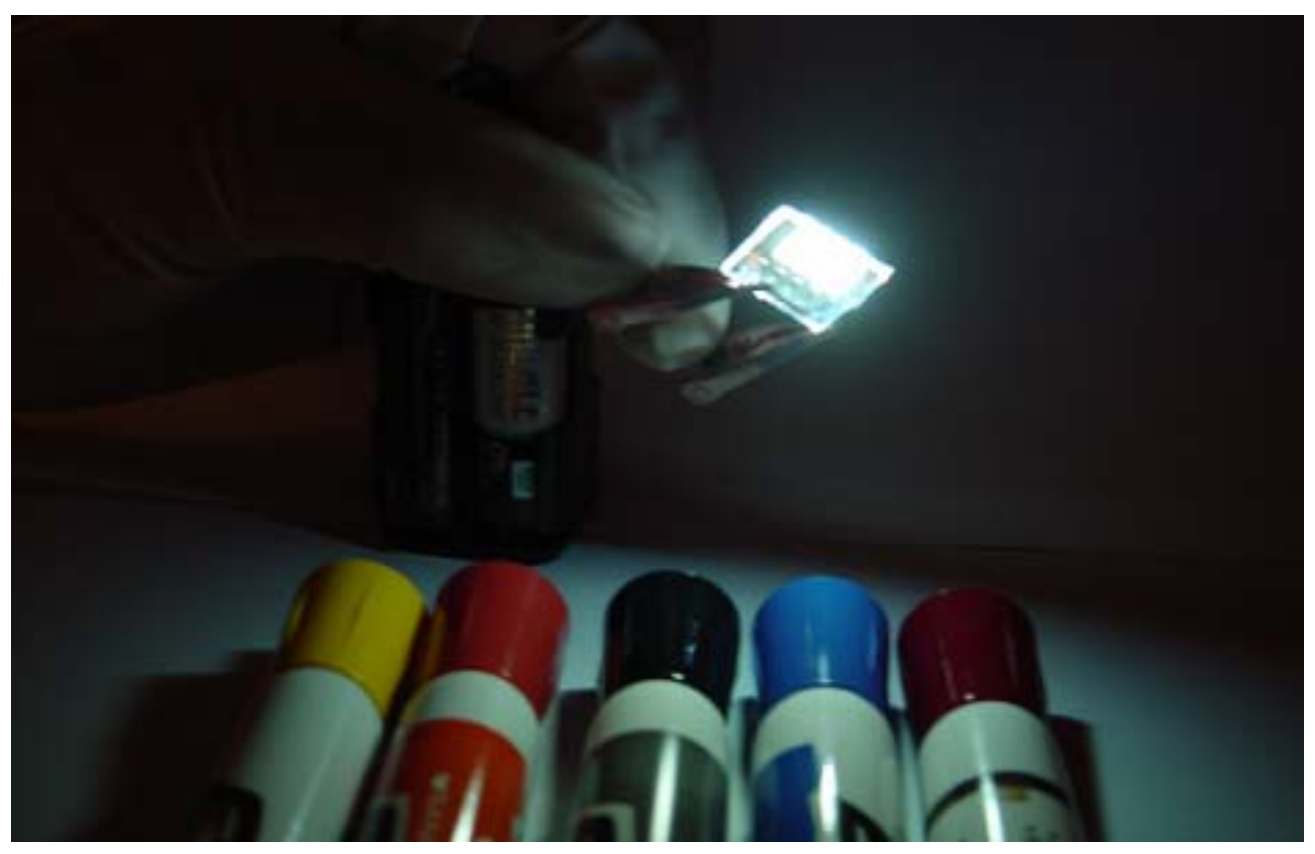

Figure 8: Agiltron's white OLED device with an excellent color rendering index of over 75.

We also measured the angular distribution of emission from the device. It is a very good Lambertion distribution, as shown in Figure 9. The external power efficiency of this device can be further enhanced by over $40 \%$ in combination with conventional light extraction approaches.

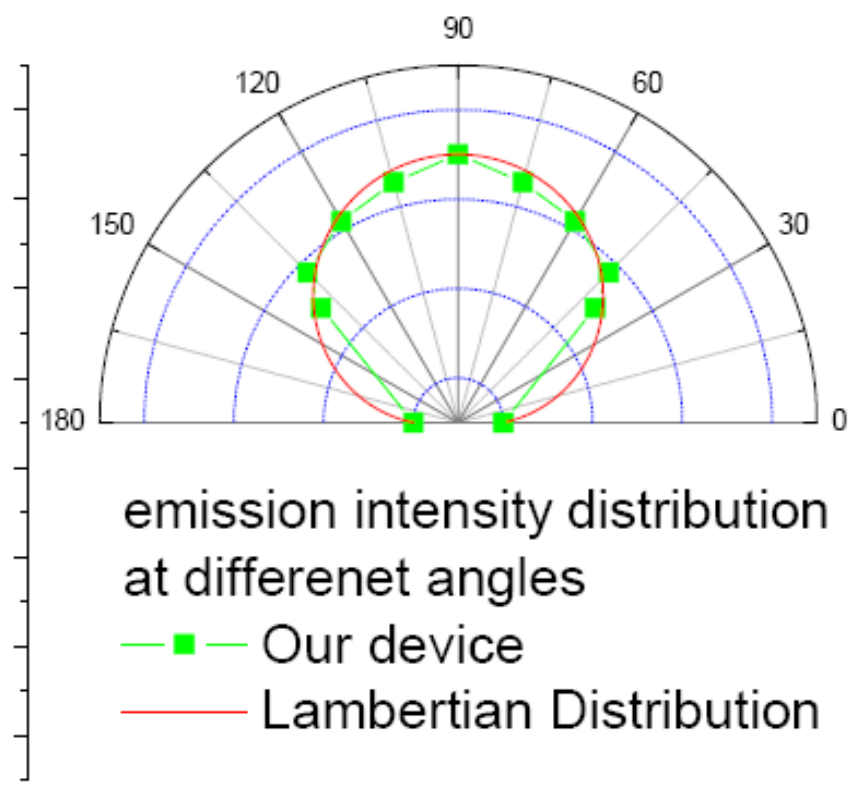

Figure 9: Emission intensity distribution of Agiltron's OLED device at different angles 
The device evaluation was performed at UCLA, whose OLED evaluation facilities were calibrated using industry standards. The light intensity at a specific current density is measured by a PR 650. Then the current-voltage-luminance curves are measured using Keithley source-measure meter together with a silicon photodiode. The device area is 12 $\mathrm{mm}^{2}$, the detector area is around $100 \mathrm{~mm}^{2}$, and the distance is $2 \mathrm{~cm}$. At UCLA they also used a directed coupled detection system, in which the detector contacts the device directly in order to collect all the light. In both our systems, device efficiency is the same with an error bar of approximately 5\%. To calibrate with industry standard, they sent our device to OSRAM and Canon, Inc to calibrate the efficiency with simple encapsulation by epoxy. Despite the possible degradation due to poor encapsulation, the efficiency from these two independent parties is the same.

\section{Mist Deposition of Nano-Particle Suspension}

We have also successfully demonstrated that the nano-particle suspension can be mist coated. The mist coating was performed at Penn State. The RMS surface roughness of the coating is $0.374 \mathrm{~nm}$. The mist coated surface is smooth enough for OLED fabrication, as shown in Figure 10.

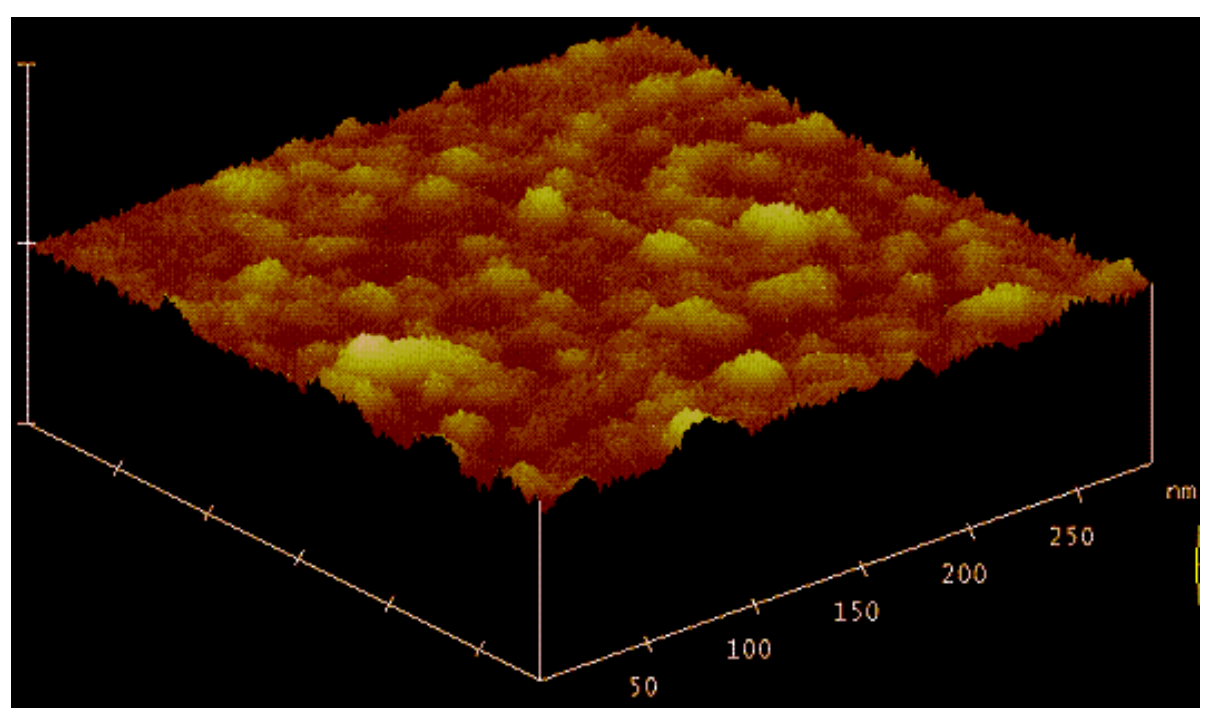

Figure 10: An AFM image of a mist coated film

\section{Future Research \& Development Areas of Interest}

This DOE Core Technology program has laid a platform for future high efficient OLED development. The following $\mathrm{R} \& \mathrm{D}$ areas of interest have been identified, which are believed to significantly help DOE to achieve its SSL goals. Agiltron is actively seeking partnerships and other funding sources (including venture capitals) to pursue these development efforts. 


\section{Area of Interest \#1: Hole transport polymers (AG I \& II) and Gold Nano- particles}

In this program, we have tried commercial PEDOT. For future research, we will try Agiltron modified TPD based hole transport materials (AG I and II) and compare their performances with that PEDOT. TPD is the mostly common hole transport material ${ }^{12345}$, and even host material ${ }^{67}$, which is being used in OLED based display products and solid state lighting source. The chemical resistivity to oxygen is not the primary concern because most organic emission materials are also oxygen sensitive, and therefore the whole device needs encapsulation eventually in the final steps. The thermal stability attracts more attention because it directly related to the lifetime of the devices. For Agiltron's hole injection layer, i. e. the cross-linking of TPD-[Si( $\left.\left(\mathrm{OCH}_{3}\right)_{3}\right]_{2}$, no thermal transitions are observed by differential scanning calotimetry in the $50 \sim 400^{\circ} \mathrm{C}$ range, indicating crosslinked TPD is extremely thermally stable. For the next step, we will also explore the air stable HIL. We have identified a highly stable hole transport materials of non-amine containing TRP to functionalize, which can work with efficient white light emissive materials to achieve highly power efficient light output.

We will also try gold NP coated substrates, and compare them with ITO NP coated substrates. The gold colloidal material is used because Au has higher work function than ITO and, which should help hole injection into active layer from ITO. It has been demonstrated a thin layer of gold $(\mathrm{Au})$ on ITO can significantly improve PLED device performance by reducing driving voltage. ${ }^{7}$ Once more, it has very good transparency when film is thin. ${ }^{8}$

\section{Area of Interest \#2: Scale up of nano-particle self-assembly process}

In this task, we will scale up the ITO nano-particle self-assembly process to 4" substrates. The nano-particle colloid preparation will be scaled up from current several milliliters to hundreds of milliliters. The coated substrates will be characterized using a sheet resistance test meter, UV-Visible spectrometer, and AFM for sheet resistance, transmittance, and surface roughness, respectively.

\footnotetext{
${ }^{1}$ Appl. Phys. Lett., 74, 24 1999, TPD derivative side-group polymers for HIL in OLED,

2 Appl. Phys. Lett.,73, 24 1998, TPD/Alq3 for hole injection layer in OLED,

3 Adv. Mater.13,1234, 2001, ITO/TPD hole injection layer in OLED,

4 Thin Solid Films, 393, 267, 2001, ITO/TPD in polymeric optical waveguide,

5 Appl. Phys. Lett., 83, 22 , 2003, TPD/NPD graded junction for OLED,

6 Thin Solid Films, 499, 306, 2006, TPD as host material for red dopant in OLED

7 Lin Ke; R.S. Kumar; Peng Chen; Lu Shen; Soo-Jin Chua; A.P. Burden, Photonics Technology Letters, IEEE, 17, 543 (2005)

${ }^{8}$ Vishal Shrotriya, Elbert Hsing-En Wu, Gang Li, Yan Yao, and Yang Yang Appl. Phys. Lett 88, 064104 (2006)
} 
In addition to the substrate size scale-up, we will extend the nano-particle selection. We will include other nano-structured materials such as high work function and highly conductive metal oxide $\left(\mathrm{V}_{2} \mathrm{O}_{5}, \mathrm{WO}_{3}\right.$ and $\left.\mathrm{MoO}_{3}\right)$. The goal of this exploration is to produce controllable hole injection composite for high power efficient OLED applications. In principle, the hole injection ability can be tuned by controlling the electrical field distribution around NPs, which is determined by the size of the NPs, electrical properties of the NPs (dielectrically constancy), and coverage of NPs on the anode. In this task, we will tune the average size and size distribution of nanoparticle, layer numbers of self-assembling, and NPs materials to realize controlled hole injection.

We will also plan to adapt this proposed nano-particle coating to industry standard automated dip coating process. The economics of this coating will be analyzed and justified for large scale commercialization.

\section{Area of Interest \#3: Evaluation of the hole injection capability of the nano-composite layer}

The hole injection capability of the nano-composite layer will be evaluated in a practical device environment using a "hole only" device geometry. The "hole only device will be fabricated using a device structure of ITO/NP/HT polymer/PF:Rubrene:PFB/Cs2CO3/Au. In this device, $\mathrm{Au}$ is used as cathode in stead of $\mathrm{Al}$. Due to the high work function of $\mathrm{Au}$, there is no electron injected from the cathode. The electrical current flowing through the device comes from the hole injected from the ITO/NP anode. Therefore, the electrical current at a define voltage reflects the hole injection capability of the anode/hole transport polymer interface. As a comparison, a control device will be fabricated using a similar device structure without the nanoparticle layer, which is ITO/HT polymer/PF:Rubrene:PFB/Cs2CO3/Au.

For the hole transport polymer, PEDOT, AG I and II, and other commercial polymer will be tested.

\section{Area of Interest \#4: OLED device fabrication, evaluation, and optimization}

The device structure will be ITO/NP/HT polymer/PF:Rubrene:ETM/Cs2CO3/Al. The device optimization is two regards. (1) optimization of the electron injection layer (Cs2CO3) to match the enhanced hole injection; (2) optimization of electron transport material (ETM) doping inside the emissive layer to enhance device power efficiency. The ETM doping can reduce turn-on voltage, control the emission zone inside the device, which increase device power efficiency.

\section{(1) Optimization of the electron injection layer}

While increasing hole injection, the electron injection also needs to catch up in order to balance hole current. As stated previously, we have already demonstrated that AG I's 
hole injection composite layer can increase hole injection. However we didn't observe significant increase of device efficiency, because increased hole injection damage the pre-optimized current balance in the device. One unique property of it is that the electron injection ability can be tuned precisely by changing the thickness of $\mathrm{Cs}_{2} \mathrm{CO}_{3}$ layer. We have very good knowledge in this technique. For the cathode side, we will use thermal evaporated or solution processed $\mathrm{Cs}_{2} \mathrm{CO}_{3}$, which has been proven to a heavily doped ntype semiconductor with super low work function of $2.2 \mathrm{eV}$. In the configuration of $\mathrm{Cs}_{2} \mathrm{CO}_{3} / \mathrm{Al}$ cathode, by change the thickness of $\mathrm{Cs}_{2} \mathrm{CO}_{3}$, we can tune the cathode work function continuously from work function of $\mathrm{Al}(4.3 \mathrm{eV})$ to around $2.2 \mathrm{eV}$, which is lower than the LUMO of most stable organic materials. Therefore, in this project, after modification of anode side with doped AG I HIL, the efficiency can be boosted by the modification of cathode side so that electron injection can catch up with increased hole injection.

\section{(2) Optimization of electron transport material (ETM) doping}

It has been proven that the hole and electron mobolities inside the emissive layer define the emission zone. Figure 11 shows several cases of distribution of emission zone under different electron, hole mobility configurations. Theoretical calculation shows that the output efficiency of PLED device can be doubled by moving the emission zone from close to the electrode (case (a) and (c)) to the middle of emission layer (case (b)) due to the microcavity effect. ${ }^{9}$ Also the efficiency loss caused by quench of exciton at metal cathode can be avoided. Here we want to adopt the material engineering solution by blend some electron/hole transport media into the host material. It will not only bring control to charge flow in the polymer layer, but also reduce device driving voltage. All these factors will significantly increase power efficiency.

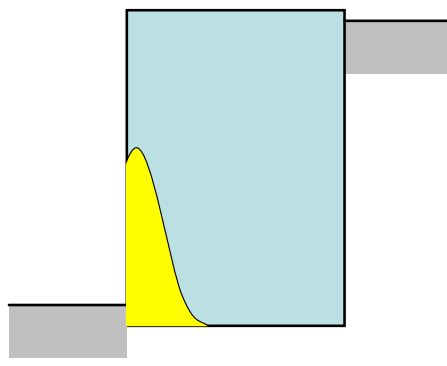

(a)

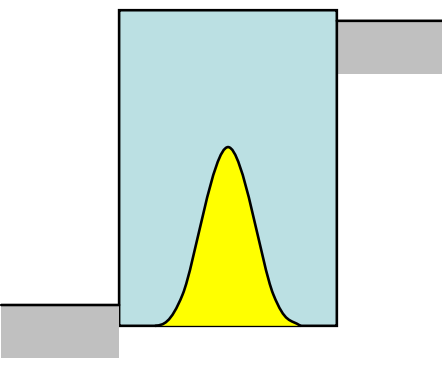

(b)

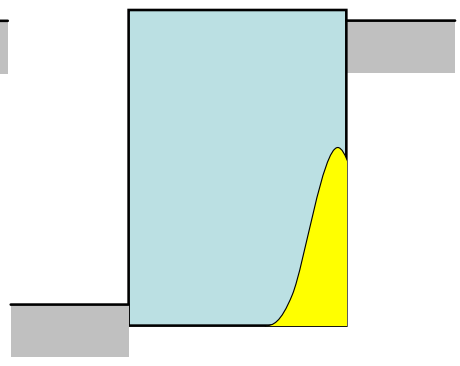

(c)

Figure 11: distribution of emission zone under different electron, hole mobility configurations: (a) electron mobility > hole mobility; (b) electron mobility = hole mobility; and (c) electron mobility < hole mobility.

The fabricated device is polyfluorene (PF) based OLED. PF has been found have much higher hole mobility than electron mobility. Therefore the recombination zone is believed to located near cathode region. Electron transport material such as PBD will be used as electron transport media. The shift of emission zone from cathode side to the middle of

\footnotetext{
${ }^{9}$ Benjamin Krummacher, Mathew K. Mathai, Vi-En Choong, Stelios A. Choulis, Franky So and Albrecht Winnacker,Organic Electronics 7, 2006, 313
} 
polymer layer can be reflected in the changed EL spectra shape due to the optical cavity effect. It is important to choose the right electron transport material in addition to its high electron transport capability, because other properties of this material such as HOMO/LUMO position, exciton lifetime have also pronounced effect on the device performance. For example, both OXD-7 and PBD are good electron transport material. So we will dope these electron transport materials in our devices to further increase the power efficiency.

\section{Area of Interest \#5: Mist coating development}

The mist coating effort of nano-particle colloids will be scaled up to 4" substrate. Initial scale up work will be done with ITO nano-particle colloids, then it will be applied to other nano-particle colloids we selected. The mist coating parameters we employed for small substrates in Year I will adapted and optimized for 4" substrate. Since organic dispersants are used for nano-particle dispersion to make colloids, post thermal treatment of mist coated films will be studied to burn out the organics. Finally the treated substrates will be characterized using a sheet resistance test meter, UV-Visible spectrometer, and AFM for sheet resistance, transmittance, and surface roughness, respectively. Preliminary device fabrication will be performed using the mist coated substrates and device performance will be compared with those on self-assembled substrates.

\section{Conclusions}

Agiltron has performed a significant amount of work in the first year of this Program and has demonstrated:

- Significant increase in hole injection/transport efficiency

- Device energy efficiency improvement

- Optimization and scale-up of air-stable and cross-linkable novel HTL nanocomposite materials synthesis

- Development of low-cost, large-scale mist deposition processes for polymer OLED fabrication

- Demonstration of how the proposed technology holds the promise to substantially improve OLED energy efficiency and lifetime

- Provided testing results showing $>27 \mathrm{lpW}$ power efficiency using the results demonstrated during this program

Agiltron is very pleased with the results demonstrated so far and looks forward to implementing these extremely important achievements in actual OLED devices in the second year of this program. 